\section{Reversing liver cirrhosis: impact of gene therapy for liver cirrhosis}

Liver cirrhosis, the irreversible end result of fibrous scarring and hepatocellular regeneration, is a major cause of morbidity and mortality worldwide with no effective therapy. Hepatic dysfunction, esophageal varices, ascites, and liver cancer are the most serious complications and are often fatal. Various factors induce liver cirrhosis: excessive alcohol intake, viral hepatitis, drug-induced hepatic injury, prolonged biliary obstruction, the late stages of some parasitic diseases, and some genetically transmitted metabolic disorders such as Wilson's disease and hemochromatosis. Although its precise etiology is not known, cirrhosis can best be defined in terms of what is pathoanatomically certain. Cirrhosis is a chronic disease of the liver in which diffuse destruction and regeneration of hepatic parenchymal cells have occurred and in which a diffuse increase in connective tissue has resulted in disorganization of the lobular and vascular architecture. ${ }^{1}$ Therefore, ideally, strategies for the treatment of liver cirrhosis should include prevention of fibrogenesis, stimulation of hepatocyte mitosis, and reorganization of tissue architecture.

We have developed a novel gene therapy approach for rat liver cirrhosis by muscle-directed gene transfer of hepatocyte growth factor (HGF). ${ }^{2}$ Studies on dimethylnitrosamine (DMN)-induced liver cirrhosis have shown that a transfection approach, utilizing the repetitive transduction of the human HGF gene in skeletal muscles using liposomes containing the hemagglutinating virus of Japan (HVJ-liposome), can achieve this in vivo. In the rat model, DMN-induced cirrhosis is characterized by the collapse of parenchymal cells and the formation of regenerative nodules separated by fibrous septa, similar to the characteristic pathological cirrhotic changes found in humans. If left untreated, the cirrhotic rats die within 7 weeks, of massive hepatic fibrosis and liver dysfunction. HGF, which was originally identified and cloned as a potent mitogen for hepatocytes, shows mitogenic and motogenic activities for a wide variety of cells. ${ }^{3}$ In addition, HGF plays an essential role in the development and regeneration of the liver, and has anti-apoptotic action on hepatocytes. It is, therefore, reasonable to utilize HGF molecules for stimulating liver regeneration in the treatment of liver cirrhosis.

In the report on the use of HGF in gene therapy, it is of particular importance that the massive periportal and centrilobular fibrosis underwent nearly total remission following repetitive gene therapy. Although the molecular mechanisms of hepatic fibrosis are not fully under- stood, several studies have demonstrated that overexpression of transforming growth factor- $\beta 1$ (TGF- $\beta 1$ ) may play a pivotal role in the progression of fibrosis. ${ }^{4}$ It has been reported that several lines of TGF- $\beta 1$ transgenic mice, which produce high plasma levels of TGF- $\beta 1$, develop liver fibrosis. TGF- $\beta 1$ induces the phenotypic transition of hepatic stellate cells to proliferating myofibroblast-like cells which enhance production of extracellular matrix components and attenuate the degradation of the extracellular matrix proteins. TGF- $\beta 1$ is also a potent growth inhibitor of epithelial and endothelial cells including hepatocytes. Furthermore, TGF- $\beta 1$ induces apoptotic cell death in hepatocytes. Conceivably, the application of a molecule that would counteract the biological activities of TGF- $\beta 1$ or suppress the expression of TGF- $\beta 1$ in the liver may have therapeutic potential for the prevention of fibrogenesis in the liver and apoptosis of hepatocytes. In DMN-induced rat liver cirrhosis, TGF$\beta 1$ expression was enhanced in the liver during progression. Of particular interest in the report of gene therapy utilizing the HGF gene is that expression of TGF- $\beta 1$ is strongly suppressed after treatment. Consequently, the transition from hepatic stellate cells to myofibroblast-like cells in the liver was inhibited. Consistent with these findings, fibrous connective tissue components in Glisson's sheath and pseudolobule formations found in the cirrhotic liver were inhibited by the gene therapy. Moreover, HGF gene therapy prevented apoptosis of hepatocytes induced by DMN administration. Transduction of the HGF gene suppressed the expression of TGF- $\beta 1$ induced by DMN. Thus, HGF gene therapy may have improved the fibrosis in the cirrhotic liver by inhibiting TGF- $\beta 1$ expression. There is still no clear understanding of how HGF suppresses TGF- $\beta 1$ expression. We investigated the inhibitory effect of recombinant HGF on the production of TGF- $\beta 1$ from cultured non-parenchymal liver cells which spontaneously produce TGF- $\beta 1$. Production of TGF- $\beta 1$ mRNA as well as TGF- $\beta 1$ protein was inhibited by HGF protein in vitro (unpublished data). Molecular mechanisms which explain how HGF suppresses expression of TGF- $\beta 1$ remain to be addressed.

In the cirrhotic rat liver, HGF stimulated hepatocyte mitosis which play an important role in progress of liver regeneration. Moreover, histopathological examination revealed that the lobular and vascular architecture was well-reorganized after gene therapy. Measurement of portal pressure revealed that portal hypertension was markedly improved after gene therapy. These findings indicate that HGF gene therapy resulted in the prevention of fibrogenesis, the stimulation of liver regeneration, and the reorganization of hepatic architecture - the key 
criteria for a potential treatment for liver cirrhosis. It is noteworthy that transfection of rats with $40 \mu \mathrm{g}$ of HGF DNA rescued all rats from fatal liver cirrhosis, and the cirrhosis was in complete remission.

Several transfection methods and vector systems have been developed to deliver exogenous genes into the liver, but all have limitations. For example, adenoviral vectors cause cytotoxicity due to the highly immunogenic properties of adenoviral proteins. Retroviral vectors have been used for gene delivery into livers in combination with a partial hepatectomy, but transfection efficiency was relatively low. Conceivably, it may not be advisable to place damaged liver cells themselves at risk of potential toxic effects of transfection. The demonstration that direct injection of liposomes containing DNA and envelope protein of HVJ into skeletal muscle is a useful way to deliver a protein to the systemic circulation constitutes an important medical advance. This method can be applied to living animals because of its simplicity, safety and lack of toxicity. Furthermore, a sustained plasma level of HGF was achieved by the repetitive transfection of the HGF gene. Repetitive in vivo transfection can be carried out without significant inflammation or activation of the cellular and humoral immune system..$^{5}$ The first successful trial to express transgenes in muscle involved the direct injection of DNA encoding various marker proteins and resulted in gene expression in muscle fibers for more than 2 months. ${ }^{6}$ Unfortunately, attempts to translate this into large animals revealed poor transfer efficiencies in comparison to those obtained in mice. Since HVJliposome-mediated gene transfer to muscle has shown much higher transfer efficiencies than those obtained by naked DNA injection, the extension of this approach to large animals should be investigated further.

Although the mechanism is not well understood, transduction of HGF gene into skeletal muscle boosts endogenous rat HGF in vivo. Gene therapy resulted in the sustained expression of plasma levels of HGF about three times as high as normal levels, as the product of both gene transfer and endogenous HGF production. HGF is a heparin-binding heterodimer related to plasminogen, and it binds avidly to heparan sulphate, a glycosaminoglycan which constructs proteoglycan with core protein.
Proteoglycans are found especially on the surface of epithelial cells including hepatocytes. Thus, HGF accumulates in the liver and is proteolytically cleaved from a single-chain precursor to generate the mature molecule.

Tumorigenicity has been reported in transgenic mice overexpressing HGF at a level more than 50-fold higher than normal mice. ${ }^{7}$ We did not find any evidence of tumor formation in rats that had received HGF gene therapy. In addition, in transgenic mice that express HGF at levels similar to those in our current experiment (two to three times higher than normal controls), the development of neoplastic tumors has been reported to be inhibited. 8 The repetitive transfection of HGF to muscle is a simple and safe method. Muscle-directed HGF gene therapy may eventually be translated into a useful clinical regimen for the treatment of patients with liver cirrhosis, which is otherwise fatal and untreatable by conventional therapy.

$\mathrm{J}$ Fujimoto ${ }^{1}$ and $\mathrm{Y}$ Kaneda ${ }^{2}$

${ }^{1}$ First Department of Surgery, Hyogo College of Medicine, Nishinomiya 663-8501, Japan; and ${ }^{2}$ Division of Gene Therapy Science, Osaka University Medical School, Japan

\section{References}

1 Conn O, Attenbury E. Cirrhosis. In: Schiff L, Schiff R (eds). Disease of the Liver, Vol 2. JB Lippencott Company: Philadelphia, 1993, Pp 875-941.

2 Ueki $\mathrm{T}$ et al. Hepatocyte growth factor gene therapy of liver cirrhosis in rats. Nature Med 1999; 5: 226-230.

3 Nakamura $T$ et al. Molecular cloning and expression of human hepatocyte growth factor. Nature 1989; 342: 440-443.

4 Border A, Noble A. Transforming growth factor $\beta$ in tissue fibrosis. New Engl J Med 1994; 331: 1286-1292.

5 Hirano $\mathrm{T}$ et al. Persistent gene expression in rat liver in vito by repetitive transfections using HVJ-liposome. Gene Therapy 1998; 5: $459-464$.

6 Wolff A et al. Direct gene transfer into mouse muscle in vitoo. Science 1990; 247: 1465-1468.

7 Takayama $\mathrm{H}$ et ol. Diverse tumorigenesis associated with aberrant development in mice overexpressing hepatocyte growth factor/scatter factor. Proc Natl Acad Sci USA 1997; 94: 701-706.

8 Santoni-Rugu $\mathrm{E}$ et al. Inhibition of neoplastic development in the liver by hepatocyte growth factor in a transgenic mouse model Proc Natl Acad Sci USA 1996; 93: 9577-9582. 